\title{
Evaluation of interfacial strength of multilayer thin films polymer by nanoindentation technique
}

\author{
Kenji INOUE*, Farid TRIAWAN**, Kazuaki INABA*, Kikuo KISHIMOTO*, \\ Masashi NISHI***, Michiyo SEKIYA***, Kunihiko SEKIDO*** and Akihito SAITOH*** \\ *Department of Transdisciplinary Science and Engineering, Tokyo Institute of Technology, \\ 2-12-1 Ookayama, Meguro-Ku, Tokyo 152-8550, Japan \\ E-mail: inaba.k.ag@m.titech.ac.jp \\ **Faculty of Engineering and Technology, Sampoerna University, \\ JIn. Raya Pasar Minggu, Kav. 16, Pancoran, Jakarta 12780, Indonesia \\ ${ }^{* \star *}$ Fuji-Susono Research Park, Canon Inc. \\ 4202, Fukara, Susono-shi, Shizuoka 410-1196, Japan
}

Received: 8 July 2018; Revised: 3 December 2018; Accepted: 24 January 2019

\begin{abstract}
Multilayer thin films polymer have been commonly used in a wide range of electronics applications, such as solar cells, photoconductors, semiconductors, and light-emitting diodes, in which failure due to delamination can occur. For evaluating and improving the quality of a multilayer thin-film product, the interfacial strength between its layers must be measured correctly and accurately. In this work, the interfacial strength of a multilayer thin-film polymer was quantified by measuring the interfacial energy release rate (interfacial fracture toughness), $G_{i}$, using the nanoindentation test technique. Three specimens of four-layer thin films were fabricated on a glass substrate. The top layer of each specimen was prepared with different thicknesses. The unloading curve technique introduced by Kozuki and Kishimoto (2010) was implemented to quantify the $G_{i}$ of each specimen. Then, crosssectional observation of the specimens was performed to confirm the layer thicknesses and to identify the delamination condition after the test. The measured $G_{i}$ tended to increase as the range between the two unloading curves used to calculate the delamination energy widened. This phenomenon is believed to be caused by the plastic energy of the deformed films during the indentation process. Finally, an empirical method for eliminating the effect of the plastic energy on the measured $G_{i}$ was proposed. Using this method, a pure $G_{i}$ value can be effectively extracted; thus, the interfacial strength of multilayer thin-film polymers can be measured correctly and accurately.
\end{abstract}

Keywords : Nanoindentation, Delamination, Multilayer films, Energy release rate, Interfacial strength

\section{Introduction}

Multilayer thin films are commonly used in a variety of electronics applications, such as solar cells, imaging elements, photoconductors, semiconductors, and light-emitting diodes. These electronic products heavily rely on the quality of their thin films and may not function well if failure due to delamination at the interface between layers occurs. To ensure that no delamination occurs in a commercial product, it is very important for the manufacturer to create strong interface bonding. Therefore, it is critical to measure and quantify the interfacial strength of multilayer thin-film products correctly and accurately (Chen et al., 2013; Budiman et al., 2017; Takahashi et al., 2016).

Several methods are available for measuring the interfacial strength of a thin film specimen, such as the peel test (Yamamoto and Wang, 2015), scratch test (Shibutani et al. 2007; Chang et al., 2008), and nanoindentation test (Toonder et al., 2001; Li and Siegmund, 2004; Chang et al., 2008; Kato et al., 2010; Kozuki and Kishimoto, 2010). The scratch test usually depends on the film thickness and is more applicable for hard film specimens, such as coating materials (Chang et al., 2008). In the peel test, it is difficult to clamp a soft thin-film specimen such as a polymer film or a brittle 
ceramic coating film. In comparison, the nanoindentation test method offers greater flexibility, as it is applicable for both soft and hard/brittle thin-film materials.

The nanoindentation test can be applied for measuring not only the mechanical properties, such as the Young's modulus (Doerner and Nix, 1986) and hardness (Oliver and Pharr, 1992), but also the interfacial strength of thin films. For example, Kozuki and Kishimoto (2010) demonstrated an experimental technique for estimating the interfacial strength of a thin-film specimen by analyzing the unloading curves of the load-displacement curve obtained from a nanoindentation test. The unloading curve can be estimated via theoretical analysis (Pharr and Bolshakov, 2002; Kozuki and Kishimoto, 2010), and then the delamination energy can be quantified according to the load-displacement relationship. A nanoindentation testing machine is usually equipped with a microscope, which allows observation of the indented area before and after the test. Delamination can be detected by analyzing this indented area after the indentation process, and the energy release rate due to delamination can be calculated as explained by Kozuki and Kishimoto (2010). This energy release rate can be regarded as the interfacial strength, as it represents the work needed to induce delamination.

In the present study, the unloading curve technique of the nanoindentation test introduced by Kozuki and Kishimoto (2010) is applied to measure the interfacial strength of three thin-films specimens made of polymer. Each specimen consists of four layers of thin films with different material properties, in which the top layer is prepared with three different thicknesses, and the location of delamination is unknown. According to the load-displacement curve of the nanoindentation test, we demonstrate how to technically measure the interfacial strength of specimens by quantifying the energy release rate of delamination. We also discuss how to eliminate the measurement error caused by plastic deformation. Hence, a correct and accurate energy release rate purely due to delamination can be obtained.

\section{Experimental procedures \\ 2.1 Specimen}

A schematic of the specimen used in the present work is shown in Fig. 1. It consisted of four layers of thin films, which are called Functioned polymer 1, 2, 3, and 4 and were fabricated above a glass substrate. Each layer was made of a different resin and prepared with a different thickness. The thickness of the glass substrate was $1 \mathrm{~mm}$, and the top layer (Functioned polymer 1) was prepared with three different film thicknesses: $h_{1}=15,10$, and $5 \mu \mathrm{m}$. To measure the actual thickness of each layer, each specimen was cut by a razor blade to observe its cross-sectional area. A microscope (KEYENCE VE-9800) was used for observation.

\begin{tabular}{|c|}
\hline Functioned polymer 1 \\
\hline Functioned polymer 2 \\
\hline Functioned polymer 3 \\
\hline Functioned polymer 4 \\
\hline Glass substrate \\
\hline
\end{tabular}

Fig. 1 Cross-sectional schematic of the laminated multilayer thin-film specimen.

To measure the Young's modulus of each film layer, a separate specimen comprising a single resin layer was prepared on a glass substrate with the following thicknesses: Functioned polymer 1, $12 \mu \mathrm{m}$; Functioned polymer 2, $4 \mu \mathrm{m}$; Functioned polymer 3, $1 \mu \mathrm{m}$; and Functioned polymer 4, 4 $\mu \mathrm{m}$. The measurement was performed via the nanoindentation test at room temperature.

\subsection{Measurement of material properties}


A nanoindentation testing machine (ENT-1100a Elionix hardness tester) was used for the experiment. The test was performed at room temperature using a Berkovich indenter. With this instrument, a microscope image can be obtained before and after the test. The instrument was load-controlled, and four different maximum loads, $P_{\text {max }}$, were applied to measure the Young's modulus of each thin-film layer, as well as the glass substrate. The measurement was repeated three times for every $P_{\max }$. The loading rate was set to be constant $(0.05 \mathrm{mN} / \mathrm{s})$ for each experiment.

The Young's modulus of each film layer was measured using a separate specimen containing a single layer only. The method of Oliver and Pharr was employed to calculate the Young's modulus according to the load-displacement curve ( $P$ - $h$ curve) of the nanoindentation test. Equation 1 shows the formula for calculating the reduced Young's modulus $E_{r}$ from the measured elastic unloading stiffness, $S$ (Oliver and Pharr, 1992). Figure 2 presents a schematic explanation.

$$
E_{r}=\frac{S}{2} \sqrt{\frac{\pi}{A_{c}}}
$$

Here, $A_{c}$ is the projected area of the elastic contact and is given as follows.

$$
A_{c}=23.95 h_{c}^{2}
$$

Here, $h_{c}$ is the vertical distance along with contact area (see Fig. 2), and $h_{c}$ can be calculated as follows:

$$
h_{c}=h_{\max }-\varepsilon \frac{P_{\max }}{S}
$$

where $h_{\max }$ is the maximum value of the displacement at $P_{\max }$ (maximum load) in the load-displacement curve, and $\varepsilon$ is a constant that depends on the indenter shape ( $\varepsilon=0.72$ for a Berkovich indenter).

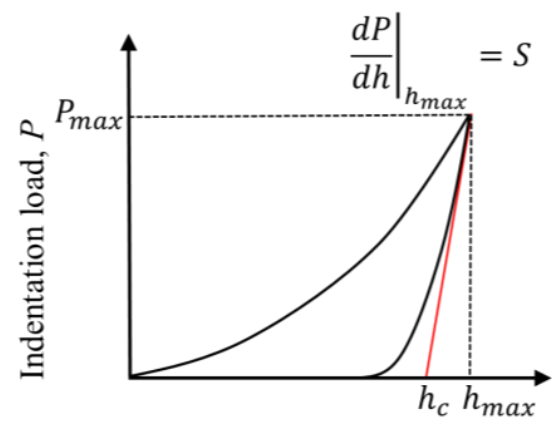

Indentation depth, $h$

Fig. 2 Load-displacement curve for calculating the Young's modulus using the measured $S$.

The measured $E_{r}$ may be affected by the deformation of the indenter. To eliminate these effects, the following equation can be used:

$$
E_{f}=\frac{1-v_{f}^{2}}{\frac{1}{E_{r}}-\frac{1-v_{i}^{2}}{E_{i}}}
$$

where $E_{f}$ and $v_{f}$ are the Young's modulus and Poisson's ratio of the specimens, respectively, and $v_{f}$ was assumed to be 0.3 in this study. $E_{i}$ and $v_{i}$ are the Young's modulus and Poisson's ratio of the indenter, respectively, which were assumed to be $1,440 \mathrm{GPa}$ and 0.07 , respectively, in this study. 


\subsection{Measurement of energy release rate of delamination}

Via the nanoindentation test, delamination in thin films can be detected by observing the area near the indented mark. A typical indentation mark on a specimen after the nanoindentation test is shown in Fig. 3. The black triangular area is the indenter mark, and the colorful area is the delamination mark, $A_{d}$. Toonder et al. (2001) found that delamination in a nanoindentation test propagated from each side of the indenter mark. A similar delamination mark was reported by Kozuki and Kishimoto (2010) in their nanoindentation test of a polyimide coating specimen. According to their surface roughness measurement, the mark was piled up, indicating delamination beneath the film.

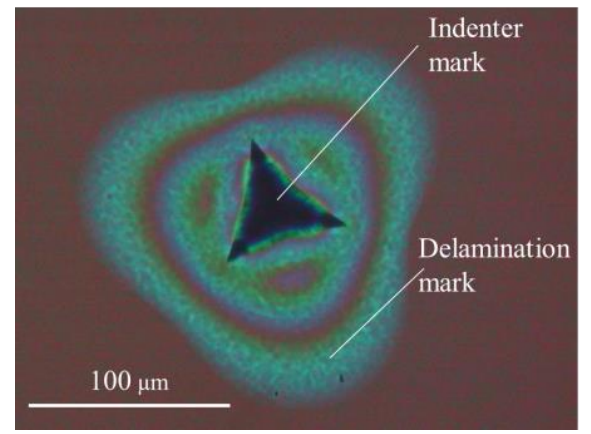

(a)

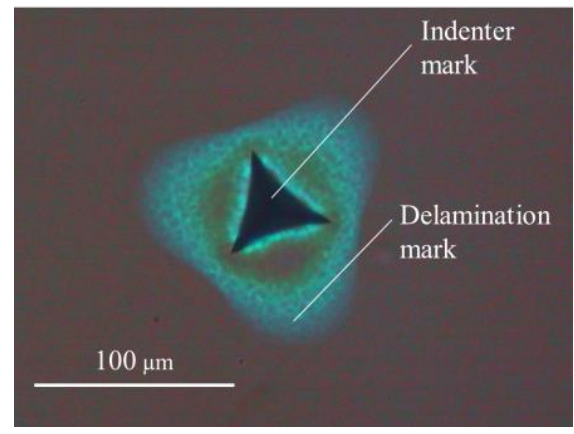

(b)

Fig. 3 Typical indentation mark after the nanoindentation test of the specimen with a $15-\mu \mathrm{m}$ top-layer thickness at (a) a maximum load of $700 \mathrm{mN}$ and (b) a maximum load of $500 \mathrm{mN}$.

A typical $P$ - $h$ curve obtained via the nanoindentation test is shown in Fig. 4 . In this case, delamination is assumed to occur at Point A and stops at Point B. Kozuki and Kishimoto (2010) proposed a method employing the unloading curves at both Points A and B to quantify the work done to generate delamination. The unloading curve can be estimated using the technique of Kozuki and Kishimoto (2010).
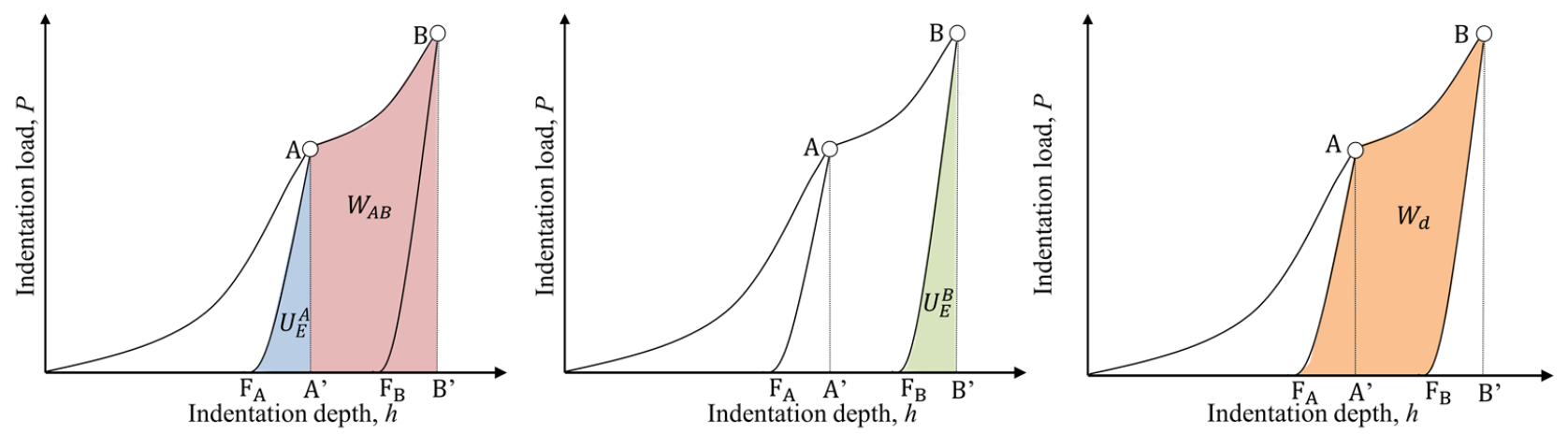

Fig. $4 P$ - $h$ curve obtained via the nanoindentation test and the related areas for deriving the delamination energy $W_{A B}$. $W_{A B}$ can be calculated from the area surrounded by $\mathrm{ABB}$ ' $\mathrm{A}^{\prime}$. $U_{E}^{A}$ can be calculated from the area surrounded by $\mathrm{AA}^{\prime} \mathrm{F}_{\mathrm{A}}$. $U_{E}^{B}$ can be calculated from the area surrounded by $\mathrm{BB}^{\prime} \mathrm{F}_{\mathrm{B}}$. The delamination energy can be determined from the area surrounded by $\mathrm{AB} \mathrm{F}_{\mathrm{B}} \mathrm{F}_{\mathrm{A}}$.

Assuming that the plastic energy due to plastic deformation and the dissipation energy caused by viscoelasticity are negligible, the delamination energy $W_{d}$ from Point A to Point B can be calculated using Eq. 5 (Omiya et al. 2008).

$$
W_{d}=U_{E}^{A}+W_{A B}-U_{E}^{B}
$$

where $W_{A B}$ is the work done by the indenter from Point $\mathrm{A}$ to Point $\mathrm{B}$, and $W_{A B}$ can be calculated from the area 
surrounded by ABB'A', as shown in Fig. 4. $U_{E}^{A}$ is the elastic energy at Point A calculated using the unloading curve of Point A, as depicted by the area surrounded by AA' $F_{\mathrm{A}} . U_{E}^{B}$ is the elastic energy calculated using the unloading curve of Point B depicted by the area surrounded by BB' $F_{\mathrm{B}}$. Using a $P$ - $h$ curve, which contains the starting and ending points of delamination (Points A and B), and the estimated unloading curves of Points A and B, the delamination energy $W_{d}$ can be quantified. $W_{d}$ is the area below the $P-h$ curve shown in Fig. 4 . Using the calculated $W_{d}$, we can calculate the corresponding energy release rate, $G_{i}$, as follows:

$$
G_{i}=\frac{W_{d}}{A_{d}}
$$

where $A_{d}$ is the delamination area obtained from the observation shown in Fig. 3. Because energy release rate, $G_{i}$, corresponds to the energy needed to generate delamination, $G_{i}$ can be considered as the interfacial strength (interfacial fracture toughness) of the thin-film specimen.

In this work, we conducted the nanoindentation test for each specimen 10-11 times with different maximum loads, $P_{\text {max }}$. This was done to divide the $P$ - $h$ curve into several unloading curves so that it could be used directly to calculate the delamination energy. A total of $10 P$ - $h$ curves with $P_{\max }$ ranging from 100 to $1,000 \mathrm{mN}$ for the specimen with $h_{1}=$ 10 and $15 \mu \mathrm{m}$ were obtained, and $11 P$ - $h$ curves with $P_{\max }$ ranging from 50 to $1,000 \mathrm{mN}$ for the specimens with $h_{1}=5$ $\mu \mathrm{m}$ were obtained.

\section{Experimental results and discussion 3.1 Young's modulus of each film layer}

The measured $E_{r}$ obtained from the unloading stiffness, $S$, of the specimen with a single film layer is plotted with respect to the indentation depth in Figs. 5(a)-(d). According to the plots, similar values of $E_{r}$ can be obtained when the indentation depth is $<10 \%$ of the film thickness. For example, the Functioned polymer 1 layer exhibited similar values of $E_{r}$ (approximately $6 \mathrm{GPa}$ ) when the indentation depth was approximately 0.4 and $0.6 \mu \mathrm{m}(<10 \%$ of the total thickness). Functioned polymer 3 exhibited similar $E_{r}$ values of approximately $5.5 \mathrm{GPa}$ at indentation depths of approximately 0.05 and $0.15 \mu \mathrm{m}$ (approximately $10 \%$ of the total thickness). Generally, the correct Young's modulus value could be obtained when the indentation depth was no more than $10 \%$ of the total film thickness, as explained by Fischer-Cripps (2001). This is likely because a measurement with a depth of $>10 \%$ could be affected by the plastic deformation or film damage during the indentation process. In this study, the Young's modulus of each film was calculated using the $E_{r}$ when the indentation depth was $<10 \%$ of the film thickness. Using Eq. 4, the average Young's modulus, $E_{f}$, of each thin-film layer and the glass substrate was obtained, and the results are presented in Table 1.

A cross-sectional image of a specimen with a thickness of $5 \mu \mathrm{m}$ for Functioned polymer 1 is shown in Fig. 6. A color change was observed in the upper part of the Functioned polymer 1 layer, which was likely due to the plastic deformation generated by the razor blade during the cutting process. According to the observation result, the thicknesses of the Functioned polymer 1 layer for the specimens were confirmed to be $h_{1}=5,10$, and $15 \mu \mathrm{m}$, and the other layer thicknesses are presented in Table 1. Furthermore, a delamination mark was observed between Functioned polymers 1 and 2, as shown in Fig. 6. That delamination was probably generated during the cutting process, as the same pattern was observed for other specimens. These results indicate that delamination caused by an indentation load was most likely to occur at the interface between Functioned polymer layers 1 and 2, as no delamination was observed at the other interfaces. That is, the delamination mark (colorful area), $A_{d}$, observed in the microscope image was likely to be the region in which delamination occurred at one single interface. Under this assumption, $A_{d}$ can be easily calculated from the observed indentation mark.

\subsection{Interfacial strength $G_{i}$}

Figure 7 shows the measured $P$ - $h$ curves for the three specimens with different thicknesses of Functioned polymer 1. The measured $P$ - $h$ curves are plotted in the same graph, thus making several unloading curves between the smallest and largest applied loads. However, it was difficult to find the starting point of delamination (Point A in Fig. 4) from the 
$P$ - $h$ curves, as no significant change was observed in the slope of the curves. The change of the slope was only observed for the specimen with $h_{1}=10 \mu \mathrm{m}$ (Fig. 7(b)); it was not observed in the $p$ - $h$ curves of other specimens.

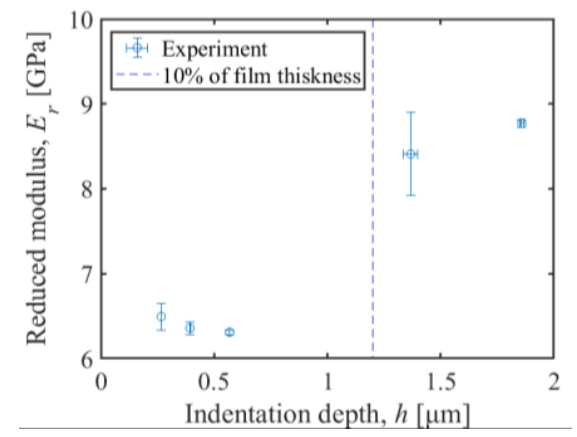

(a)

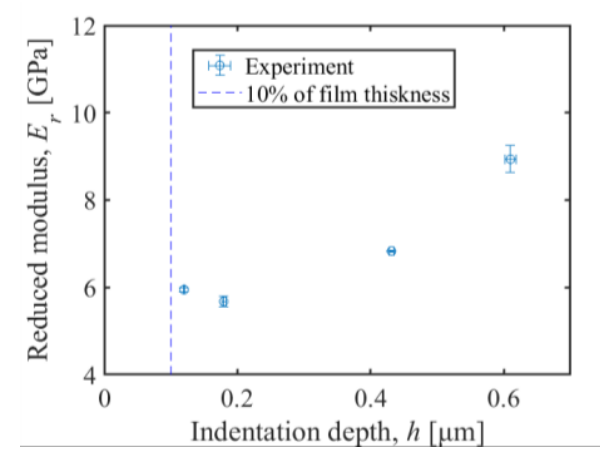

(c)

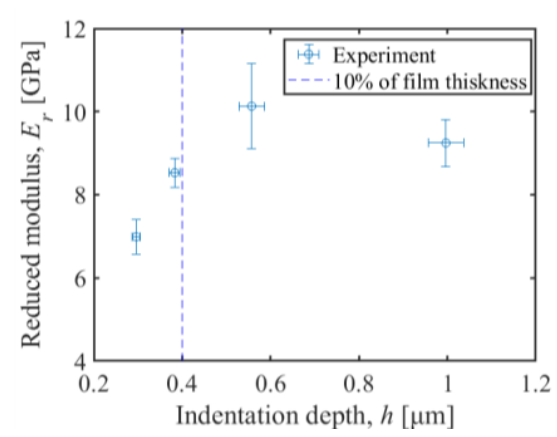

(b)

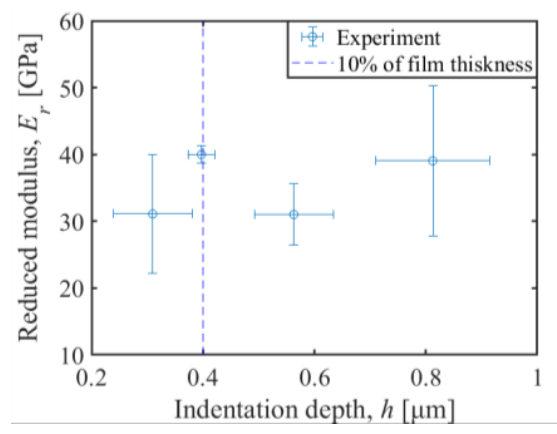

(d)

Fig. 5 Plot of the reduced modulus $E_{r}$ vs. the indentation depth for (a) Functioned polymer 1 (film thickness $12 \mu \mathrm{m}$ ), (b) Functioned polymer 2 (film thickness $4 \mu \mathrm{m}$ ), (c) Functioned polymer 3 (film thickness $1 \mu \mathrm{m}$ ), and (d) Functioned polymer 4 (film thickness $4 \mu \mathrm{m}$ ). The black dotted line indicates the indentation depth at $10 \%$ of the film thickness.

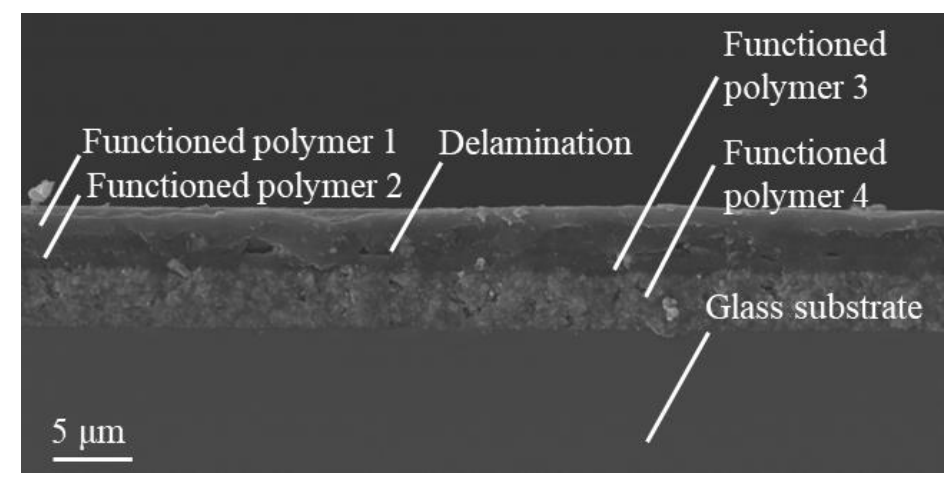

Fig. 6 Cross-sectional observation of the specimen with a Functioned polymer 1 thickness of $h_{1}=$

$5 \mu \mathrm{m}$. Delamination is likely to occur between Functioned polymer layers 1 and 2.

Table 1 Specimen specifications

\begin{tabular}{|l|c|c|c|c|}
\hline Base materials & Young's modulus $(\mathrm{GPa})$ & \multicolumn{3}{|c|}{ Thickness $(\mu \mathrm{m})$} \\
\hline Functioned polymer 1 & $6 \pm 0.1$ & $15 \pm 2$ & $10 \pm 1$ & $5 \pm 0.2$ \\
\hline Functioned polymer 2 & $7.5 \pm 1$ & $1 \pm 0.1$ & $1 \pm 0.1$ & $1 \pm 0.1$ \\
\hline Functioned polymer 3 & $5.5 \pm 0.2$ & $1 \pm 0.1$ & $1 \pm 0.1$ & $1 \pm 0.1$ \\
\hline Functioned polymer 4 & $30 \pm 10$ & $10 \pm 1$ & $2.5 \pm 1$ & $3.5 \pm 1$ \\
\hline Glass substrate & $72 \pm 0.2$ & 1,000 & 1,000 & 1,000 \\
\hline
\end{tabular}




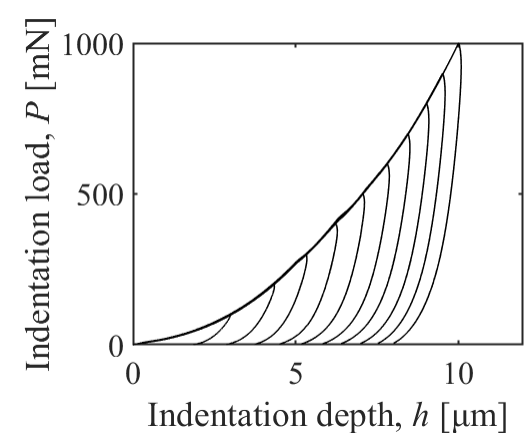

(a)

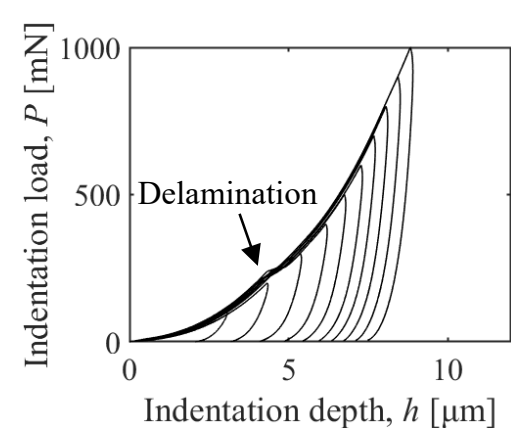

(b)

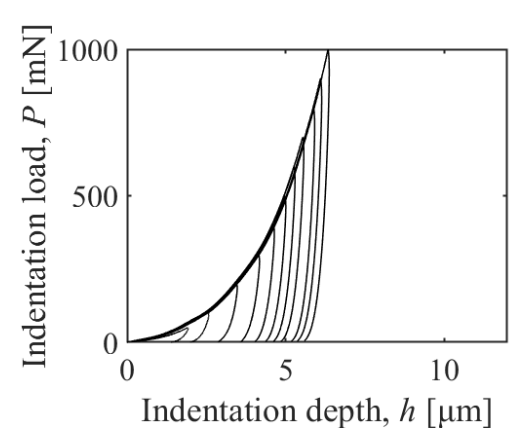

(c)

Fig. $7 P$ - $h$ curve of specimen with (a) $h_{1}=15 \mu \mathrm{m}$, (b) $h_{1}=10 \mu \mathrm{m}$, and (c) $h_{1}=5 \mu \mathrm{m}$. A change of the slope was observed at $h_{1}=10 \mu \mathrm{m}$.

Figure 8 shows the indentation marks of all three specimens when delamination was about to begin. The indentation marks are indicated by a color change. For the specimen with $h_{1}=15 \mu \mathrm{m}$, the color change was observed at $P_{\max }=$ $400 \mathrm{mN}$ (see Fig. 8(a)). For the specimen with $h_{1}=10 \mu \mathrm{m}$, the delamination began at $P_{\max }=300 \mathrm{mN}$, and for the specimen with $h_{1}=5 \mu \mathrm{m}$, the delamination began at $P_{\max }=100 \mathrm{mN}$.

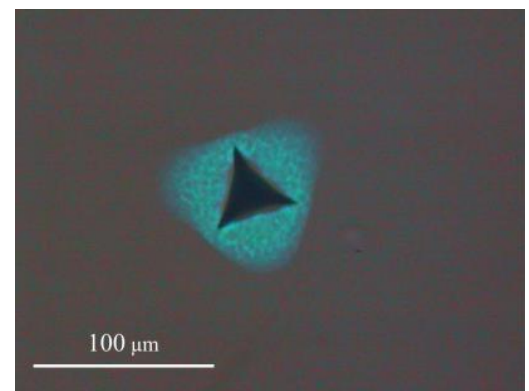

(a)

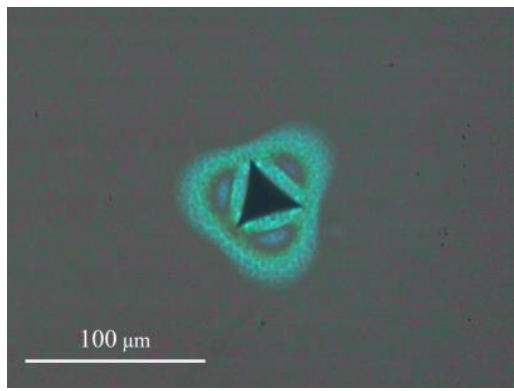

(b)

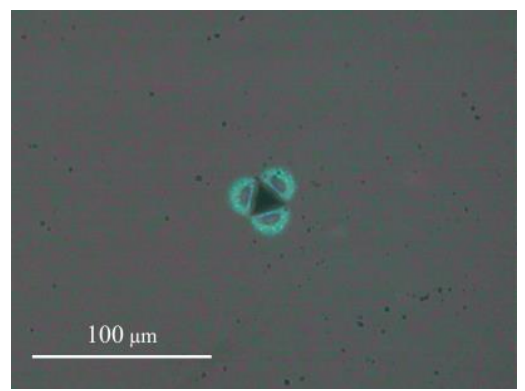

(c)

Fig. 8 Indentation marks when delamination began to propagate for specimens with (a) $h_{1}=15 \mu$ m, max load $P_{\max }=$ $400 \mathrm{mN}$; (b) $h_{1}=10 \mu \mathrm{m}$, max load $P_{\max }=300 \mathrm{mN}$; and (c) $h_{1}=5 \mu \mathrm{m}$, max load $P_{\max }=100 \mathrm{mN}$.

To determine the exact starting point of delamination, the delamination area was plotted with respect to the indentation depth, as shown in Fig. 9, for the three specimens. The delamination area increased linearly with the increase of the indentation depth. This means that the intersection point between the horizontal axis and the fitting line can be considered as the starting point of delamination. Therefore, we can conclude that the starting loads of delamination for the specimens with $h_{1}=15,10$, and $5 \mu \mathrm{m}$ were 344,230 , and $64.5 \mathrm{mN}$, respectively.

In a nanoindentation test with a Berkovich indenter, the unloading curves are usually well-described by the power law (Pharr and Bolshakov, 2002), as follows:

$$
P=\alpha\left(h-h_{f}\right)^{2}
$$

where $h_{f}$ is the final displacement after complete unloading. The constant $\alpha$ is a material constant. Figure 10 plots $h_{f}$ and $\alpha$ against $P_{\max }$ for $h_{1}=10 \mu \mathrm{m}$. $h_{f}$ and $\alpha$ increased linearly with respect to $P_{\max }$. Thus, the $h_{f}$ and $\alpha$ at the starting point of delamination can be determined from the plot in Fig. 10.

Figure 11 shows the $P-h$ curve with the estimated unloading curve at $h_{1}=10 \mu \mathrm{m}$. As shown, the estimated unloading curve fit the experimental result well. Regarding the starting point of delamination, the point estimated in Fig. 9 is almost the same as the point at which the slope of the $P$ - $h$ curve changes. This indicates that the unloading curve was well-estimated and can be used to calculate the delamination energy. 


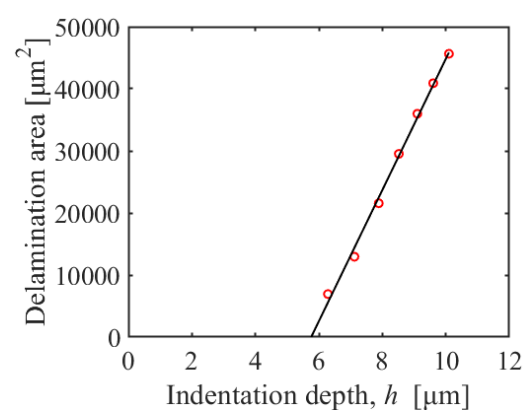

(a)

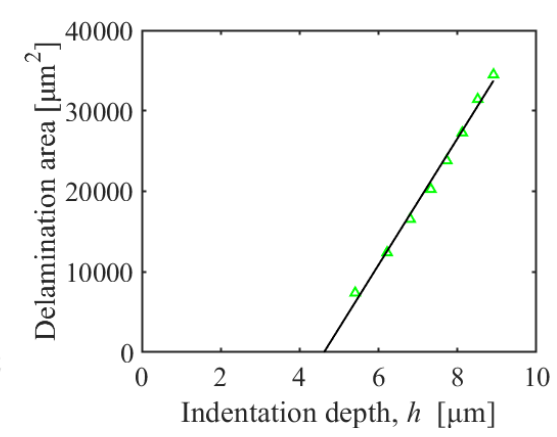

(b)

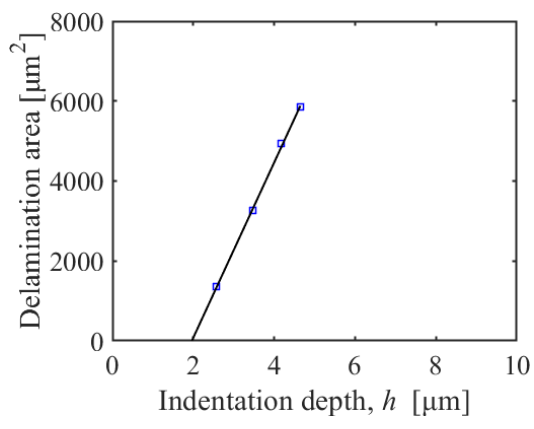

(c)

Fig. 9 Delamination area with respect to the indentation depth $h$ for (a) $h_{1}=15 \mu \mathrm{m}$, (b) $h_{1}=10 \mu \mathrm{m}$, and (c) $h_{1}=5 \mu \mathrm{m}$.

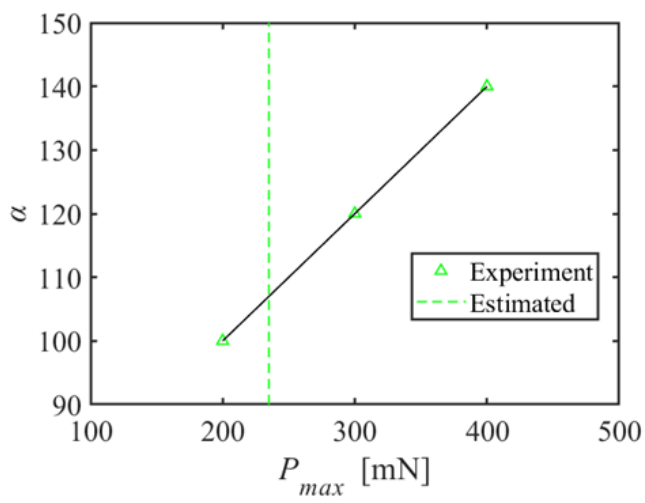

(a)

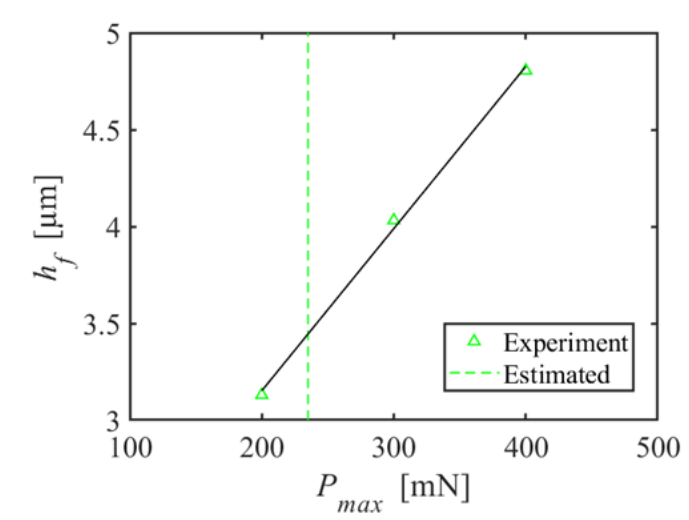

(b)

Fig. 10 Relationships of (a) $\alpha$ and (b) $h_{f}$ with $P_{\max }$ for $h_{1}=10 \mu \mathrm{m}$. These parameters increased linearly with respect to $P_{\max }$. The black dotted line indicates the indentation load at the estimated starting point of delamination.

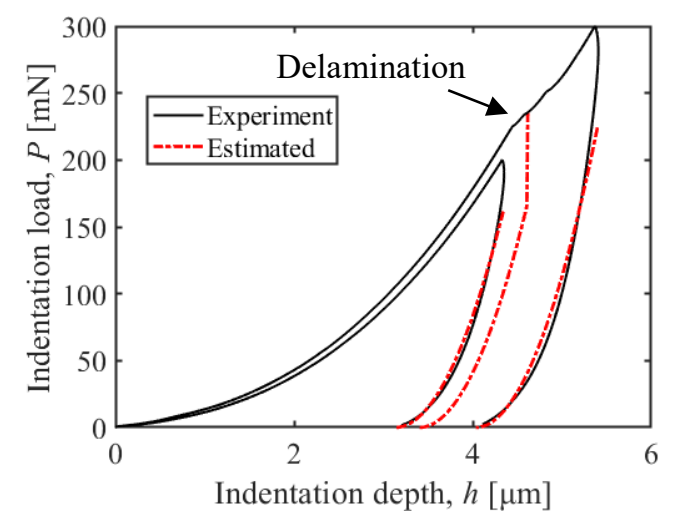

Fig. 11 Experimental $P$ - $h$ curve, along with the estimated unloading curve, for the specimen with $h_{1}=10 \mu \mathrm{m}$.

The $G_{i}$ values calculated at different ranges of the indentation load, $\Delta P$, are presented in Table 2 for the specimen with $h_{1}=15 \mu \mathrm{m}$, Table 3 for the specimen with $h_{1}=10 \mu \mathrm{m}$, and Table 4 for the specimen with $h_{1}=5 \mu \mathrm{m}$. According to these results, the energy release rate tended to increase as $\Delta P$ increased. 
Table 2 Energy release rate of the specimen with $h_{1}=15 \mu \mathrm{m}$

\begin{tabular}{|l|l|l|l|l|l|l|l|}
\hline Ending point of delamination* $(\mathrm{mN})$ & 400 & 500 & 600 & 700 & 800 & 900 & 1,000 \\
\hline Interfacial strength $G_{i}\left(\mathrm{~J} / \mathrm{m}^{2}\right)$ & 26 & 39 & 43 & 45 & 46 & 51 & 54 \\
\hline
\end{tabular}

*The starting point of delamination is assumed to be $344 \mathrm{mN}$.

Table 3 Energy release rate of the specimen with $h_{1}=10 \mu \mathrm{m}$

\begin{tabular}{|l|l|l|l|l|l|l|l|l|}
\hline Ending point of delamination* $(\mathrm{mN})$ & 300 & 400 & 500 & 600 & 700 & 800 & 900 & 1,000 \\
\hline Interfacial strength $G_{i}\left(\mathrm{~J} / \mathrm{m}^{2}\right)$ & 30 & 39 & 45 & 50 & 54 & 59 & 63 & 68 \\
\hline
\end{tabular}

*The starting point of delamination is assumed to be $230 \mathrm{mN}$.

Table 4 Energy release rate of the specimen with $h_{1}=5 \mu \mathrm{m}$

\begin{tabular}{|l|l|l|l|l|l|}
\hline Ending point of delamination* $(\mathrm{mN})$ & 100 & 200 & 300 & 400 & 500 \\
\hline Interfacial strength $G_{i}\left(\mathrm{~J} / \mathrm{m}^{2}\right)$ & 38 & 53 & 70 & 86 & 95 \\
\hline
\end{tabular}

*The starting point of delamination is assumed to be $64.5 \mathrm{mN}$.

The increased $G_{i}$ indicates that energy other than the elastic energy was used during the nanoindentation test. Most likely, this energy was generated by the plastic deformation or was dissipation energy caused by the viscoelasticity of the films during the indentation process, because the value tended to increase with the increasing load. This additional energy became more obvious as the thickness of Functioned polymer 1 decreased (Table 4), and it was negligible at a large thickness (Table 2).

To check whether the viscoelasticity affected the results, the $P$ - $h$ curves with three dufferent loading rates for the specimen with $h_{1}=15 \mu \mathrm{m}$ were obtained, as shown in Fig. 12. As shown in Fig. 12(b), as the loading rate increased, the indentation load increased at the same indentation depth. However, in this experiment, the delamination occurred when the indentation load was between 300 and $400 \mathrm{mN}$, and a very small difference was observed between the three curves, as shown in Fig. 12(a). This indicates that viscoelasticity of the specimen did not significantly affect the delamination during the experiment.

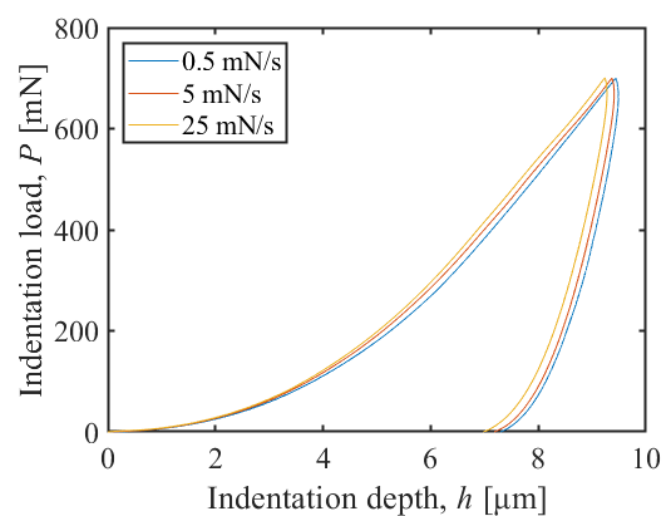

(a)

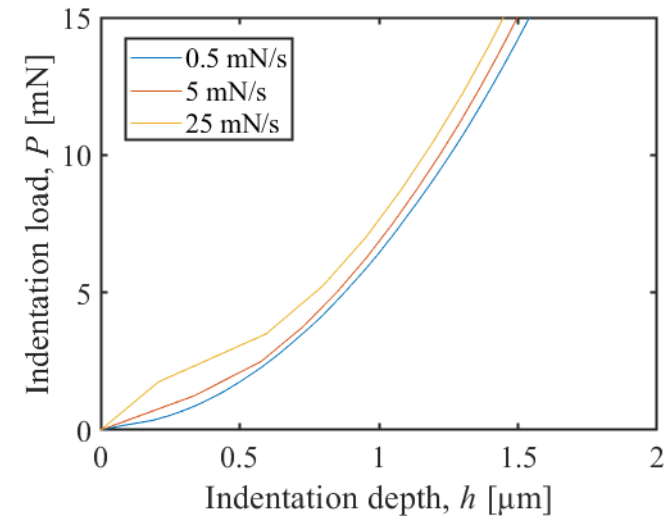

(b)

Fig. $12 P$ - $h$ curves with three different loading rates: (a) overall view and (b) around the starting point of loading. There is a small difference when the indentation load is $>300 \mathrm{mN}$.

Figure 13 shows a plot of the energy release rate $G_{i}$ and the range of load $\Delta P$ applied in the calculation (Tables 2-4) for all the specimens. A curve fitting line was implemented for each plot in order to make an extrapolation line for $\Delta P$ close to zero. The three curve fitting lines met at the same intersection point. The results indicate that the influence of the plastic deformation can be omitted; thus, a pure $G_{i}$ could be obtained. From the plot, it can be concluded that the interfacial strength of the specimens was approximately $28 \mathrm{~J} / \mathrm{m}^{2}$. The good agreement among the three specimens indicates that the proposed method is valid, as it does not depend on the thickness. Therefore, the interfacial strength can be judged as correct and accurate. 


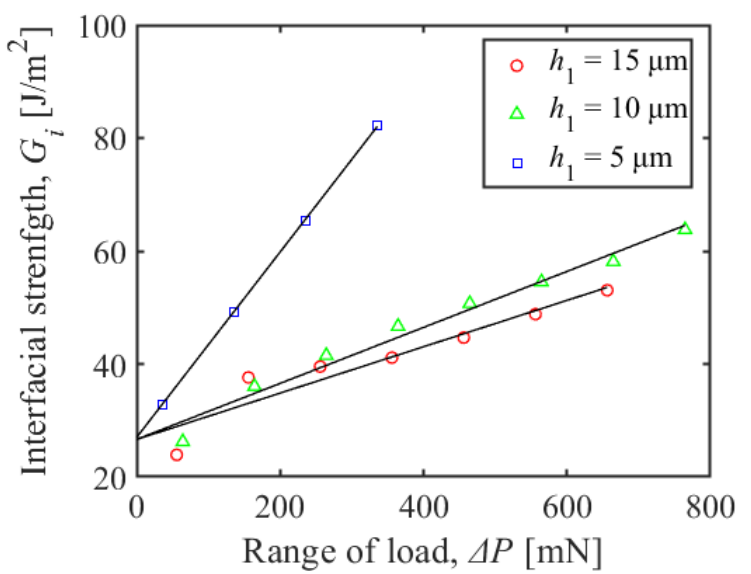

Fig. 13 Plot of the measured interfacial strength $G_{i}$ vs. the range of load $\Delta P$ with the fitting lines made at each Functioned polymer 1 thickness $h_{1}$. For each $h_{1}, G_{i}$ shows a similar value at $\Delta P=0$.

\section{Conclusion}

The nanoindentation test technique was utilized to evaluate the interfacial strength of multilayer thin-film polymer specimens. Three specimens made of the same materials with four-layer films were fabricated on a glass substrate. The top layer of each specimen was prepared with a different thickness. Prior to measuring the interfacial strength, the Young's modulus of each film layer was measured via the nanoindentation test using the method of Oliver and Pharr. Cross-sectional observation was performed to confirm the thickness of each layer and to identify the location of delamination. The Young's moduli of the four layers and the glass substrate were identified, and the thickness of each film was confirmed. Delamination caused by the nanoindentation test was likely to occur at the interface between the $1^{\text {st }}$ and $2^{\text {nd }}$ layers. The interfacial strength was measured by quantifying the energy release rate, $G_{i}$ (interfacial fracture toughness), using the unloading curve method of the nanoindentation test introduced by Kozuki and Kishimoto (2010). Several unloading curves were created with a small range of load $\Delta P$, from which the delamination energy was calculated. To precisely calculate the delamination energy, the starting point of delamination was determined from the plot of the delamination area with respect to the indentation depth, as well as experimental results in which the delamination occurrence was used as the ending point of delamination. $G_{i}$ tended to increase with $\Delta P$. This was attributed to the effects of plastic energy or dissipation energy due to the viscoelasticity of the films generated during the indentation process. An empirical method was employed to eliminate these effects on the measured $G_{i}$. This was done by plotting the $G_{i}$ and the corresponding $\Delta P$ of all specimens with different thicknesses. The pure $G_{i}$ could be obtained when $\Delta P$ was close to zero, where the plastic energy is considered negligible. Using this procedure, we determined that the interfacial strength, $G_{i}$, of the multilayer thin-film polymer specimen was approximately $28 \mathrm{~J} / \mathrm{m}^{2}$. This value is independent of the film thickness and therefore can be judged as correct and accurate.

\section{References}

Budiman, B. A., Triawan, F., Adziman, F. and Nurprasetio, I. P., Modeling of stress transfer behavior in fiber-matrix composite under axial and transverse loadings, Composite Interfaces, Vol. 24., No.7 (2017), pp. 677-690.

Chang, S. Y., Tsai, H. C., Chang, J. Y., Lin, S. J. and Chang, Y. S., Analyses of interface adhesion between porous SiOCH low-k film and SiCN layers by nanoindentation and nanoscratch tests, Thin Solid Films, Vol. 516, No. 16 (2008), pp. 5334-5338.

Chen, C., Shishido, N., Koiwa, K., Kamiya, S., Sato, H., Nishida, M., Omiya, M., Suzuki, T., Nakamura, T., Suzuki, T. and Nokuo, T., Specimen size effect of interface strength distribution induced by grain structure of $\mathrm{Cu}$ line, Transactions of the Japan Society of Mechanical Engineers Part A, Vol. 79, No. 799 (2013), pp. $354-358$ (in Japanese).

Doerner, M. F. and Nix, W. D., A Method for interpreting the data from depth-sensing indentation instruments, Journal of Materials Research, Vol. 1, No. 4 (1986), pp. 601-609. 
Fischer-Cripps, A., Nanoindentation (2001), p103, Springer-Verlag.

Kato, M., Fujioka, K., Akebono, H. and Sugeta, A., Measurement of delamination energy of sputtered SiC film coated on tool steel substrate with conical precipitates by micro edge-indent method, Journal of JSEM, Vol. 10, Special Issue (2010), pp. 141-145.

Kozuki, K. and Kishimoto, K., Film thickness dependency of interfacial strength evaluation for thin film coating structure by nanoindentation method, Journal of Solid Mechanics and Materials Engineering, Vol. 4, No. 6 (2010), pp. 771 780 .

Li, W. and Siegmund, T., An analysis of the indentation test to determine the interface toughness in a weakly bonded thin film coating - substrate system, Acta Materialia, Vol. 52, No. 10 (2004), pp. 2989-2999.

Oliver, W. C. and Pharr, G. M., An improved technique for determining hardness and elastic modulus using load and displacement sensing indentation experiments, Journal of Materials Research, Vol. 7, No. 6 (1992), pp. 1564-1583.

Omiya, M., Kishimoto, K. and Nakano, T., Evaluation of the interfacial strength of layered structures by indentation method, Journal of Applied Mechanics, Vol. 75, No. 3 (2008), 031006.

Pharr, G. M. and Bolshakov, A., Understanding nanoindentation unloading curves, Journal of Materials Research, Vol. 17, No. 10 (2002), pp. 2660-2671.

Shibutani, T., Yu, Q., Shiratori, M. and Akai, T., Mechanism of damage process on $\mathrm{Si}_{3} \mathrm{~N}_{4} / \mathrm{Cu}$ interface in nanoscratch test, Journal of Solid Mechanics and Materials Engineering, Vol. 1, No. 3 (2007), pp. 322-331.

Takahashi, Y., Aihara, K., Ashida, I., Higuchi, K., Yamamoto, Y., Arai, S., Muto, S. and Tanaka, N., Evaluation of interfacial fracture strength in micro-components with different free-edge shape, Bulletin of the JSME, Vol.3, No.6 (2016), pp. 1-10.

Toonder, J. D., Malzbender, J., With, G. D. and Balkenende, R., Fracture toughness and adhesion energy of sol-gel coatings on glass, Journal of Materials Research, Vol. 17, No. 1 (2002), pp. 224-233.

Wang, H. and Yamamoto, A., Peel force of electrostatic adhesion in crawler-type electrostatic climbing robots, Journal of the Japan Society of Applied Electromagnetics and Mechanics, Vol. 23, No. 3 (2015), pp. 498-503. 